\title{
28 Research Soure \\ Early Changes in Gene Expression Profiles in AML Patients During Induction Chemotherapy
}

\author{
Ingrid Jakobsen \\ Linköping University \\ Max Sundkvist \\ Linköping University \\ Niclas Björn \\ Linköping University \\ Henrik Gréen \\ Linköping University \\ Kourosh Lotfi ( $\nabla$ kourosh.lotfi@liu.se) \\ Linköping University Hospital
}

\section{Research Article}

Keywords: chemotherapy, AML patients, differential gene expression (DGE), pathway analysis, NF-KB signaling.

Posted Date: August 5th, 2021

DOI: https://doi.org/10.21203/rs.3.rs-643327/v2

License: (c) (i) This work is licensed under a Creative Commons Attribution 4.0 International License. Read Full License 


\section{Abstract}

Background: Elucidation of the genetic mechanisms underlying treatment response to standard induction chemotherapy in AML patients is warranted, in order to aid in risk-adapted treatment decisions as novel treatments are emerging. In this pilot study, we explored the treatment-induced expression patterns in a small cohort of AML patients by analyzing differential gene expression (DGE) over the first two days of induction chemotherapy.

Methods: Blood samples were collected from ten AML patients at baseline (before treatment initiation) and during the first two days of treatment (Day 1; approximately $24 \mathrm{~h}$, and Day 2; approximately $48 \mathrm{~h}$ after treatment initiation, respectively) and RNA was extracted for subsequent RNA sequencing. DGE between time points were assessed by pairwise analysis using the $\mathrm{R}$ package edgeR version 3.18.1 in all patients as well as in relation to treatment response (complete remission, CR, vs non-complete remission, $\mathrm{nCR}$ ). Ingenuity Pathway Analysis (Qiagen) software was used for pathway analysis and visualization.

Results: After initial data quality control, two patients was excluded from further analysis, resulting in a final cohort of eight patients with data from all three timepoints. DGE analysis demonstrated activation of pathways with genes directly or indirectly associated with NF-KB signaling. Significant activation of the NF-KB pathway was seen in $50 \%$ of the patients two days after treatment start, while iNOS pathway effects could be identified already after one day. nCR patients displayed activation of pathways associated with cell cycle progression, oncogenesis and anti-apoptotic behavior, including the STAT3 pathway and Salvage pathways of pyrimidine ribonucleotides. Notably, a significant induction of cytidine deaminase, an enzyme responsible for the deamination of Ara-C, could be observed between baseline and Day 2 in the nCR patients but not in patients achieving CR.

Conclusions: In conclusion, we show that time-course analysis of gene expression represents a feasible approach to identify relevant pathways affected by standard induction chemotherapy in AML patients. This poses as a potential method for elucidating new drug targets and biomarkers for categorizing disease aggressiveness and evaluating treatment response. However, more studies on larger cohorts are warranted to elucidate the transcriptional basis for drug response.

\section{Background}

The standard induction chemotherapy in acute myeloid leukemia (AML) have remained roughly the same over the last decades, with treatment consisting of the nucleoside analog cytarabine (Ara-C) in combination with an anthracycline, most commonly daunorubicin or idarubicin. Additional drugs have been used together with the cornerstone drugs in different clinical trials, and in specific cases such as that of acute promyelocytic leukemia, treatments targeting specific chromosomal rearrangements are available (1). However, AML patients still suffer from a significant degree of therapyrelated mortality and treatment failure. More knowledge is needed of the genetic mechanisms underlying poor treatment response in $\mathrm{AML}$, in order to achieve better risk stratification and individual adaption of treatment regimens as newer drugs are emerging in clinical trials. Genetic variation in genes encoding drug-metabolizing enzymes, transporters, DNArepair systems or in genes regulating cell cycle progression and apoptosis is likely influencing cellular drug response both in leukemic and non-leukemic cells, although the clinical utility of such markers in relation to AML treatment decisions remain controversial (2). In leukemia, gene expression profiling of diagnostic samples has been implicated as a tool able to discriminate between different types (e.g ALL and AML) (3) as well as molecular and prognostic subclasses of AML (4-6). It has also been shown that acute lymphoblastic leukemia cells of different molecular subtypes share common but treatment-specific acute gene expression changes induced by mono- or combination therapy, as measured by gene expression arrays of samples collected before and one day after treatment (7). In addition, a recent study of normal p53regulated apoptosis, induced by DNA damage, demonstrated that the extent of the apoptotic response was attributable to 
the rapidity of the transcriptional response rather than to a specific set of target genes (8). This shows that time series analysis of gene expression could provide new insights to the mechanisms behind drug response. The early drug-induced gene expression changes in AML patients undergoing standard induction chemotherapy has, to our knowledge, not been investigated previously. In this pilot study, we aimed to explore the expression patterns in a small cohort of AML patients and analyzing differential gene expression over the first two days of induction chemotherapy. Using RNA sequencing data, the changes in gene expression over time was analyzed overall to identify common patterns, but also compared between patients achieving complete remission and those who did not, to identify potential genes and pathways that might be used to predict or understand the response.

\section{Materials And Methods}

\section{Patients and sample collection}

10 patients with AML scheduled for induction chemotherapy at the hematology clinic of Linköping University Hospital were included in the study. The study was performed in compliance with the Declaration of Helsinki under the approval by the local ethics committee (Forsknings etikkomittén vid Hälsouniversitet Linköping University, Sweden), and all patients gave informed consent. Patient characteristics and treatment details are summarized in Table 1. Blood samples were collected at baseline (before treatment initiation) and during the first two days of treatment (Day 1; approximately $24 \mathrm{~h}$ after treatment initiation, and Day 2; approximately $48 \mathrm{~h}$ after treatment initiation). All patients received Ara-C either as $100 \mathrm{mg} / \mathrm{m}^{2}$ as a continuous infusion (Patient 2) and Patient 3) or as $1 \mathrm{~g} / \mathrm{m}^{2}$ twice daily as $2 \mathrm{~h}$ infusions before the Day 1 sample collection; before the Day 2 sample collection all patients except Patient 2 and Patient 3 had also received anthracycline treatment either as idarubicin $\left(10 \mathrm{mg} / \mathrm{m}^{2}\right.$ as $1 \mathrm{~h}$ infusion; Patient 1 and Patient 4$)$ or daunorubicin $\left(60 \mathrm{mg} / \mathrm{m}^{2}\right.$ as $8 \mathrm{~h}$ infusion). Patient 4 was also part of a randomized phase 2 trial investigating the addition of cladribine, as a $5 \mathrm{mg} / \mathrm{m}^{2} 1 \mathrm{~h}$ infusion two times, to Ara-C and idarubicin (9). Patient 5 was initially suspected to have acute promyelocyte leukemia and received treatment with all-trans retinoic acid (ATRA) before the final AML diagnosis and inclusion in this study. In addition to chemotherapy, the patients received standard supportive care with common comedications including dexamethasone eye drops, allopurinol and antiemetics. RNA was extracted from EDTA blood samples using the Qiagen Blood RNA mini kit (Qiagen), or from blood samples stabilized in PAXgene blood RNA tubes (PreAnalytiX) using the PAXgene blood RNA kit (PreAnalytiX), according to the manufacturers' instructions. The RNA concentration was measured on a NanoDrop (Thermo Scientific) and the RNA integrity number (RIN) was assessed using an Agilent 2100 BioAnalyzer (Agilent) with the Agilent RNA 6000 nano kit (Agilent) according to the manufacturer's instructions.

\section{Sequencing and data preprocessing}

All samples with a RIN > 7.5 were sent to the National Genomics Infrastructure (NGI, Stockholm, Sweden) hosted by the Science for Life Laboratory, for RNA sequencing. Libraries were prepared with the TruSeq RNA Library Prep Kit v2 (Illumina) with poly-A selection and paired-end (2x100bp) sequencing was performed on the NovaSeq 6000 Sequencing System (Illumina) using an s1 flow cell. Two samples, Patient 6 Day 2 and Patient 10 Day 2, failed library preparation and due to the low amount of extracted RNA no further attempts could be made. Preprocessing of the raw sequencing data was performed by NGI according to the $\mathrm{nf}$-core/rnaseq pipeline (see https://github.com/nf-core/rnaseq for details). Briefly, the pipeline processes the raw fastq data using FastQC for read quality control and TrimGalore! for adapter removal and quality trimming. Alignment of the reads to the human reference genome GRCh38 was made using STAR version 2.7.2b (10). The read distributions over genomic features were summarized using featureCounts from the Subread package version 1.6.2 (11, 12), and additional quality-control of the results was performed using DupRadar (13) for duplication rate quality control, edgeR $(14,15)$ for sample similarity, Preseq $(16)$ for library complexity estimation, and 
RSeQC (17) or read distribution, duplication and gene body coverage. Finally, the quality measures were summarized using MultiQC version 1.7 (18).

\section{Differential gene expression analysis}

To explore changes in gene expression in response to treatment, R version 3.6.1 (19) and the R package edgeR version 3.18.1 $(14,15)$ was used. The data was structured to contain read counts, ensemble gene ID, patient ID and sample time point, for all genes with read counts $>1$ count per million (CPM) in $\geq 3$ samples. The data was then normalized using the calcNormFactors to scale the raw library sizes for each sample. Differential gene expression (DGE) compared to baseline levels were investigated by pairwise comparisons. In the analysis of DGE between time points, were replicates were lacking for individual patients, the biological coefficient of variation (BCV) was defined as 0.4 as recommended in the edgeR user guide. Negative binomial generalized linear models (GLMs) were fitted for each patient using the function GLMFit with the dispersion defined as the BCV squared. Likelihood ratio tests were then performed with the function glmLRT to determine which genes were differentially expressed between the time points. For the overall analysis, the function estimateDisp was used to determine the trended, tagwise and common dispersion for the samples. These were then fitted using gImQLFit and a quasi-likelihood F-test was performed using glmQLFTest. Gene expression changes were also investigated in the clinical response groups, morphological complete remission $(\mathrm{CR} ; \mathrm{n}=5)$ and non-complete remission ( $\mathrm{nCR} ; \mathrm{n}=5$ ) as assessed by bone marrow sampling between day 15 and day 28 after treatment initiation.

\section{Pathway analysis}

The function TopTags in edgeR was used to extract differentially expressed (DE) genes and their $\log _{2}$ (fold change), $p$ value and false discovery rate adjusted $p$-value. DE genes with $p$-values $\leq 0.05$ were used as input for Ingenuity Pathway Analysis (IPA) (QIAGEN) software that analyses and visualizes DE genes to identify activated or inhibited pathways (20). Canonical pathways extracted from IPA with a - $\log (p$-value) $\geq 1.3$ (corresponding to a p-value $<0.05)$ were marked as inhibited if the Z-score was $\leq-2$ and activated if it was $\geq 2$. All pathway images in the manuscript were generated using IPA (QIAGEN). Molecules are represented as nodes, and the biological relationship between two nodes is represented as an edge (line). All edges are supported by at least one literature reference or canonical information stored in the IPA knowledge base. The intensity of the node color indicates the degree of up- or down-regulation in red and green, respectively. Nodes are displayed using various shapes that represent the functional class of the gene product. Edges are displayed with various labels that describe the nature of the relationship between the nodes (e.g., $\mathrm{P}$ for phosphorylation, T for transcription). 
Table 1

AML patient characteristics.

\begin{tabular}{|c|c|c|c|c|c|c|c|c|}
\hline $\begin{array}{l}\text { Patient } \\
\text { ID }\end{array}$ & $\begin{array}{l}\text { Year of } \\
\text { diagnosis }\end{array}$ & Age & $\begin{array}{l}\text { Induction } \\
\text { treatment } \\
\text { regime }\end{array}$ & Cytogenetics & $\begin{array}{l}\text { FLT3 } \\
\text { ITD }\end{array}$ & $\begin{array}{l}\text { NPM1 } \\
\text { mutation }\end{array}$ & $\begin{array}{l}\text { CEBPA } \\
\text { mutation }\end{array}$ & $\begin{array}{l}\text { Morphological } \\
\text { complete } \\
\text { remission } \\
\text { after } \\
\text { induction } 3\end{array}$ \\
\hline 1 & 2001 & 67 & $\begin{array}{l}\text { Idarubicin } \\
\text { and } \\
\text { cytarabine }\end{array}$ & $\begin{array}{l}\text { Aberrant } \\
\text { expression } \\
\text { of T-cell } \\
\text { markers }\end{array}$ & NA & NA & NA & No \\
\hline 2 & 2001 & 81 & $\begin{array}{l}\text { Daunorubicin } \\
\text { and } \\
\text { cytarabine }^{1}\end{array}$ & NA & NA & NA & NA & Yes \\
\hline 3 & 2003 & 39 & $\begin{array}{l}\text { Daunorubicin } \\
\text { and } \\
\text { cytarabine }^{1}\end{array}$ & $\begin{array}{l}\text { Normal } \\
\text { karyotype }\end{array}$ & No & No & NA & Yes \\
\hline 4 & 2001 & 67 & $\begin{array}{l}\text { Cladribine, } \\
\text { cytarabine } \\
\text { and } \\
\text { idarubicin }\end{array}$ & Trisomy 8 & NA & NA & NA & Yes \\
\hline 5 & 2003 & 66 & $\begin{array}{l}\text { Daunorubicin } \\
\text { and low-dose } \\
\text { cytarabine }^{2}\end{array}$ & $\begin{array}{l}1- \\
40 \mathrm{dmin}[22] \\
/ 46 \mathrm{XY}[3]\end{array}$ & Yes & No & NA & No \\
\hline 6 & 2015 & 64 & $\begin{array}{l}\text { Daunorubicin } \\
\text { and } \\
\text { cytarabine }\end{array}$ & $\begin{array}{l}\text { Normal } \\
\text { karyotype }\end{array}$ & No & Yes & Heterozygous & Yes \\
\hline 7 & 2015 & 74 & $\begin{array}{l}\text { Daunorubicin } \\
\text { and } \\
\text { cytarabine }\end{array}$ & $\begin{array}{l}\text { Not possible } \\
\text { due to no } \\
\text { mitoses } \\
\text { after } \\
\text { culturing }\end{array}$ & NA & NA & NA & No \\
\hline 8 & 2015 & 73 & $\begin{array}{l}\text { Daunorubicin } \\
\text { and } \\
\text { cytarabine }\end{array}$ & $\begin{array}{l}\text { Normal } \\
\text { karyotype }\end{array}$ & Yes & No & No & No \\
\hline 9 & 2016 & 21 & $\begin{array}{l}\text { Daunorubicin } \\
\text { and } \\
\text { cytarabine }\end{array}$ & $\begin{array}{l}47 X Y \\
\text { inv(16) } \\
\text { (p13;q22), } \\
+ \text { mar[23] }\end{array}$ & No & No & NA & Yes \\
\hline 10 & 2016 & 46 & $\begin{array}{l}\text { Daunorubicin } \\
\text { and } \\
\text { cytarabine }\end{array}$ & $\begin{array}{l}\text { Complex } \\
\text { karyotype }\end{array}$ & No & No & No & No \\
\hline
\end{tabular}

\section{Results}

\section{Sequencing data quality assessment}

Based on high duplication rate, skewed GC distribution and large portion of multimapping reads, Patient 6 and 10 were excluded from further analysis. The skewed GC distribution and high levels of overrepresented sequences are indicative 
of ribosomal, bacterial or viral RNA contamination, or biased selection during sample preparation. It could be noted that both these patients had extremely high expression of one single gene - $H B B$ - encoding the hemoglobin subunit $\beta$ hemoglobin, with feature counts of several million. However, due to the overall quality metrics divergences, the decision to exclude these two patients remained. This resulted in a final cohort of eight patients with data from all three time points available.

\section{Common changes in gene expression}

Pairwise comparisons with the assessment of overlap between individual patients, and analysis of DGE overall between the time points of the whole cohort demonstrated significant activation of pathways with genes directly or indirectly associated with NF-KB signaling. This includes processes regulating immune responses, including inflammatory processes, proteasomal processing, lymphogenesis and cell survival as well as proliferation (21). In addition, iNOS production is associated with NF-KB signaling, and this could be observed in our data set as DGE between time points in the iNOS pathway (Fig. 1 and Fig. 2 illustrate the significant transcriptional changes observed in the NF- $K B$ pathway and iNOS pathway, respectively). The entire list of affected pathways with Z-scores can be found in Additional file 1, Table S1 (individual patient data) and Additional file 2, Table S2 (response groups and Overall data). Significant activation of the NF-KB pathway was seen in $50 \%$ of the patients two days after treatment start, while iNOS pathway effects could be identified already after one day.

\section{Pathways affected in relation to treament outcome}

We also investigated which pathways were affected during the treatment in relation to patient outcome; $\mathrm{CR}$ or $\mathrm{nCR}$, based on day 15 or day 28 bone marrow evaluation. In patients reaching $\mathrm{CR}$, the pathway Oxidative phosphorylation was activated already after the first treatment day, while the pathways Purine de novo biosynthesis II, tRNA charging, and Cyclins and cell cycle regulation were inhibited. These pathways were not significant in patients that did not reach CR. In contrast, nCR patients displayed activation of pathways associated with cell cycle progression, oncogenesis and antiapoptotic behavior, including activation of the STAT3 pathway (Fig. 3) and Salvage pathways of pyrimidine ribonucleotides (Fig. 4). Notably, a significant induction of cytidine deaminase, an enzyme responsible for the deamination of Ara-C, could be observed between baseline and Day 2 in the nCR patients but not in the patients achieving CR. All pathways affected with their corresponding z-scores in the two response groups are listed in Additional file 2, Table S2.

\section{Discussion}

In this pilot study, we analyzed gene expression changes over the first two days of standard induction chemotherapy in AML patients. We could show induction of relevant pathways related to immune response, cell proliferation and apoptosis, but also including genes of importance for drug pharmacokinetics. Some of these changes were detectable already after the first day of treatment. Overall, the NF-KB pathway was significantly activated after two days of treatment compared to baseline. This was also reflected by expression alterations in the iNOS pathway, which is directly linked to NF-KB signaling. The canonical NF-KB pathway is normally activated by pro-inflammatory cytokines, Toll-like receptor binding or T-cell receptor activation, subsequently leading to proteasomal degradation of the NF-KB inhibitor IкB. The release from IKB allows NF-kB to relocate from the cytoplasm to the nucleus where it regulates a wide variety of genes. In oncogenesis, this includes the transcription of genes promoting cell proliferation and survival as well as angiogenesis, metastasis, and suppression of the tumorsuppressors p53 and FOXO3a (21). In AML, a growing body of evidence has demonstrated dysregulated NF-KB (22) and opened up the possibility of targeting this pathway; here, proteasome inhibitors are constituting a promising group of drugs with broad application to many types of cancers. In addition, in vitro experiments have shown that treatment with cytarabine induces NF-KB expression in the HL-60 cell line (23). Our results in contrast show relative up-regulation of IKB and correspondingly down-regulation of NF-KB between 
baseline and Day 2. This is indicative of an overall positive effect of the chemotherapy treatment, inhibiting cell proliferation and survival; however, we cannot determine whether these transcriptional changes are present in all cell populations (malignant as well as healthy) or to which extent these relative changes are reflecting a decreasing proportion of malignant cells (with assumed dysregulated NF-KB signaling).

When analyzing the patients based on their first response evaluation (bone marrow analysis at day 15 or 28), we were able to identify pathways that were significantly affected in one group but not the other. These differences could potentially be indicative of differences in tumor cell sensitivity to drug treatment. As such, the pathways Purine de novo biosynthesis II, tRNA charging, and Cyclins and cell cycle regulation were inhibited in patients reaching CR already after the first course of induction therapy, which might demonstrate reduced tumor cell proliferation in this group. These pathways were not significantly affected in the group of patients that did not achieve complete remission; however, two patients did so after additional treatment. In contrast to the CR group, nCR patients displayed significant activation of pathways associated with cell cycle progression, anti-apoptotic signaling and malignant transformation. Persistent activation of Stat3, as well as other Stat proteins, has been implicated as an oncogene promoting malignant transformation in various experimental settings $(24,25)$. Stat 3 target genes include $\mathrm{Bcl}-\mathrm{X}$ and $\mathrm{Bcl}-2$, both encoding antiapoptotic proteins, and cooperation with c-Jun constitute yet another mechanism of interference with tumor cell apoptosis. In addition, the targets Cyclin D1 and Myc promote proliferation; in solid tumors an influence of Stat3 on VEGF expression could also further promote tumor growth and metastasis/tissue invasion through increased angiogenesis, a process of lower relevance for blood cancers. Many other target genes are likely involved in the oncogenic potential of Stat3, and activation of this protein does not necessarily lead to malignant transformation. However, suppression of Stat signaling has demonstrated tumor growth arrest, induction of apoptosis in cancer cells and inhibition of malignant transformation, indicating that it has potential as a future drug target $(24,26-28)$. Our results suggest that patients displaying rapid activation of the Stat3 pathway could be harboring more resistant or highly proliferative leukemic clones. Interestingly, another pathway identified to be significantly activated in patients with poor initial treatment response was Salvage pathways of pyrimidine ribonucleotides. This pathway represents an important system for the maintenance of ribonucleotide pools in all proliferating cells, but also have implications for drug response (29, 30). Notably, the induction of the enzyme cytidine deaminase (CDA) could result in a more treatment-resistant phenotype in these patients, as the pyrimidine analog Ara-C is subject to degradation into Ara-U by CDA. CDA induction as a mechanism of resistance to Ara-C has been demonstrated in vitro $(31,32)$, and our results support also an in vivo relevance.

Some considerations of this study need to be addressed. Even though our study population is small and the influence of interindividual variation may occlude common changes in gene expression related to the treatment, we are partly compensating for this by comparing the different time point data to that from the baseline sample for each patient. However, the bulk RNA sequencing data represent the gene expression in both healthy and cancerous blood cells, and the leukemic blast proportion in blood samples from AML patients varies. Throughout the treatment, blood leukocyte counts are rapidly decreasing in many patients, and in our approach, we are not discriminating between cancer cells and healthy cells being eradicated. Changes in the relative abundance of different cell populations are likely to be reflected in the expression data. As an example, we cannot determine whether a decreased gene expression at day two compared to baseline means that the expression has been inhibited, or if it means that a specific cell population responsible for the baseline expression was eradicated, leaving only cell populations with another pattern. In addition, our patient samples were collected over a long period, and variations in treatment protocols and sampling procedures may have influenced the result. Initial principal component analysis indicated that the data tended to partly cluster depending on inclusion period (defined as before or after the introduction of national treatment guidelines, data not shown) although there was an overlap between these clusters. 
In conclusion, we show that time-course analysis of gene expression represents a feasible approach to identify relevant pathways affected by standard induction chemotherapy in AML patients. This poses as a potential method for elucidating new drug targets and biomarkers for categorizing disease aggressiveness and evaluating treatment response. However, more studies on larger cohorts are warranted to elucidate the transcriptional basis for drug response. As single-cell RNA sequencing methodologies are developed and becoming more common, this would likely represent an option to further enhance the resolution also in time course studies like this one, although single-cell approaches are associated with other practical challenges in both sample management and analysis compared to conventional bulk RNA sequencing. Preferably, future studies would include more time points as well as follow-up analysis in the event of relapse.

\section{Declarations}

\section{Ethics approval}

The study was performed in compliance with the Declaration of Helsinki under the approval by the local ethics committee (Forskningsetikkomittén vid Hälsouniversitet Linköping University, registration numbers 98319, 02-459 and 2017-397-31), and all patients gave informed consent to study participation and publication of the study results.

\section{Data availability}

The sequencing datasets generated and/or analysed during the current study are not publicly available due to restraints of the ethical permit, but are available from the corresponding author on reasonable request.

\section{Declaration of conflicts of interest}

The authors declare no competing interests. The research grant providers had no role in the study design, collection, analyses or interpretation of data, writing of the manuscript, or in the decision to publish the results.

\section{Funding}

This study was funded by grants from The Swedish Cancer Society (HG), Lions research fund for noncommunicable diseases (IJ), and ClasGroschinsky Memorial Fund (HG).

\section{Authors' contributions}

HG: conception and study design, sample preparation and analysis; IJ: sample preparation, clinical data compilation, results interpretation, and manuscript preparation; MS and NB: bioinformatics and statistical analysis, manuscript preparation; KL: Patient recruitment, sample collection and clinical data collection. All authors critically reviewed the manuscript and approved the final version for publication.

\section{Acknowledgements}

We gratefully acknowledge the invaluable help from lab technicians Lucia Pelle (Ph.D), Kerstin Willander (Ph.D) and Naomi Yamada (research engineer) in the sample collection process. The authors also wish to acknowledge NBIS (National Bioinformatics Infrastructure Sweden). The authors acknowledge sequencing support from the National Genomics Infrastructure in Genomics Production Stockholm funded by Science for Life Laboratory, the Knut and Alice Wallenberg Foundation and the Swedish Research Council. Parts of the sequencing data computations and handling were enabled by resources provided by the Swedish National Infrastructure for Computing (SNIC) at Uppsala Multidisciplinary Center for Advanced Computational Science (UPPMAX) partially funded by the Swedish Research Council through grant agreement no. 2018-05973. 


\section{References}

1. Dohner H, Estey E, Grimwade D, Amadori S, Appelbaum FR, Buchner T, et al. Diagnosis and management of AML in adults: 2017 ELN recommendations from an international expert panel. Blood. 2017;129(4):424-47.

2. Megias-Vericat JE, Montesinos P, Herrero MJ, Boso V, Martinez-Cuadron D, Poveda JL, et al. Pharmacogenomics and the treatment of acute myeloid leukemia. Pharmacogenomics. 2016;17(11):1245-72.

3. Golub TR, Slonim DK, Tamayo P, Huard C, Gaasenbeek M, Mesirov JP, et al. Molecular classification of cancer: class discovery and class prediction by gene expression monitoring. Science. 1999;286(5439):531-7.

4. Bullinger L, Dohner K, Bair E, Frohling S, Schlenk RF, Tibshirani R, et al. Use of gene-expression profiling to identify prognostic subclasses in adult acute myeloid leukemia. The New England journal of medicine. 2004;350(16):160516.

5. Lindvall C, Furge K, Bjorkholm M, Guo X, Haab B, Blennow E, et al. Combined genetic and transcriptional profiling of acute myeloid leukemia with normal and complex karyotypes. Haematologica. 2004;89(9):1072-81.

6. Schoch C, Kohlmann A, Schnittger S, Brors B, Dugas M, Mergenthaler S, et al. Acute myeloid leukemias with reciprocal rearrangements can be distinguished by specific gene expression profiles. Proc Natl Acad Sci U S A. 2002;99(15):10008-13.

7. Cheok MH, Yang W, Pui CH, Downing JR, Cheng C, Naeve CW, et al. Treatment-specific changes in gene expression discriminate in vivo drug response in human leukemia cells. Nat Genet. 2003;34(1):85-90.

8. Bainer RO, Trendowski MR, Cheng C, Pei D, Yang W, Paugh SW, et al. A p53-regulated apoptotic gene signature predicts treatment response and outcome in pediatric acute lymphoblastic leukemia. Cancer Manag Res. 2017;9:397-410.

9. Juliusson G, Hoglund M, Karlsson K, Lofgren C, Mollgard L, Paul C, et al. Increased remissions from one course for intermediate-dose cytosine arabinoside and idarubicin in elderly acute myeloid leukaemia when combined with cladribine. A randomized population-based phase II study. British journal of haematology. 2003;123(5):810-8.

10. Dobin A, Davis CA, Schlesinger F, Drenkow J, Zaleski C, Jha S, et al. STAR: ultrafast universal RNA-seq aligner. Bioinformatics. 2013;29(1):15-21.

11. Liao Y, Smyth GK, Shi W. featureCounts: an efficient general purpose program for assigning sequence reads to genomic features. Bioinformatics. 2014;30(7):923-30.

12. Liao Y, Smyth GK, Shi W. The Subread aligner: fast, accurate and scalable read mapping by seed-and-vote. Nucleic Acids Res. 2013;41(10):e108.

13. Sayols S, Scherzinger D, Klein H. dupRadar: a Bioconductor package for the assessment of PCR artifacts in RNA-Seq data. BMC Bioinformatics. 2016;17(1):428.

14. Robinson MD, McCarthy DJ, Smyth GK. edgeR: a Bioconductor package for differential expression analysis of digital gene expression data. Bioinformatics. 2010;26(1):139-40.

15. McCarthy DJ, Chen Y, Smyth GK. Differential expression analysis of multifactor RNA-Seq experiments with respect to biological variation. Nucleic Acids Res. 2012;40(10):4288-97.

16. Daley T, Smith AD. Predicting the molecular complexity of sequencing libraries. Nat Methods. 2013;10(4):325-7.

17. Wang L, Wang S, Li W. RSeQC: quality control of RNA-seq experiments. Bioinformatics. 2012;28(16):2184-5.

18. Ewels P, Magnusson M, Lundin S, Kaller M. MultiQC: summarize analysis results for multiple tools and samples in a single report. Bioinformatics. 2016;32(19):3047-8.

19. RCoreTeam. R: A Language and Environment for Statistical Computing. R Foundation for Statistical Computing, Vienna, Austria; 2021. 
20. Kramer A, Green J, Pollard J, Jr., Tugendreich S. Causal analysis approaches in Ingenuity Pathway Analysis. Bioinformatics. 2014;30(4):523-30.

21. Wong ET, Tergaonkar V. Roles of NF-kappaB in health and disease: mechanisms and therapeutic potential. Clin Sci (Lond). 2009;116(6):451-65.

22. Zhou J, Ching YQ, Chng WJ. Aberrant nuclear factor-kappa B activity in acute myeloid leukemia: from molecular pathogenesis to therapeutic target. Oncotarget. 2015;6(8):5490-500.

23. Strum JC, Small GW, Pauig SB, Daniel LW. 1-beta-D-Arabinofuranosylcytosine stimulates ceramide and diglyceride formation in HL-60 cells. J Biol Chem. 1994;269(22):15493-7.

24. Bromberg J. Stat proteins and oncogenesis. J Clin Invest. 2002;109(9):1139-42.

25. Levy DE, Lee CK. What does Stat3 do? J Clin Invest. 2002;109(9):1143-8.

26. Kube D, Holtick U, Vockerodt M, Ahmadi T, Haier B, Behrmann I, et al. STAT3 is constitutively activated in Hodgkin cell lines. Blood. 2001;98(3):762-70.

27. Epling-Burnette PK, Liu JH, Catlett-Falcone R, Turkson J, Oshiro M, Kothapalli R, et al. Inhibition of STAT3 signaling leads to apoptosis of leukemic large granular lymphocytes and decreased Mcl-1 expression. J Clin Invest. 2001;107(3):351-62.

28. Turkson J, Ryan D, Kim JS, Zhang Y, Chen Z, Haura E, et al. Phosphotyrosyl peptides block Stat3-mediated DNA binding activity, gene regulation, and cell transformation. J Biol Chem. 2001;276(48):45443-55.

29. Frances A, Cordelier P. The Emerging Role of Cytidine Deaminase in Human Diseases: A New Opportunity for Therapy? Mol Ther. 2020;28(2):357-66.

30. Nygaard P. On the role of cytidine deaminase in cellular metabolism. Adv Exp Med Biol. 1986;195 Pt B:415-20.

31. Neff T, Blau CA. Forced expression of cytidine deaminase confers resistance to cytosine arabinoside and gemcitabine. Exp Hematol. 1996;24(11):1340-6.

32. Schroder JK, Kirch C, Flasshove M, Kalweit H, Seidelmann M, Hilger R, et al. Constitutive overexpression of the cytidine deaminase gene confers resistance to cytosine arabinoside in vitro. Leukemia. 1996;10(12):1919-24.

\section{Figures}




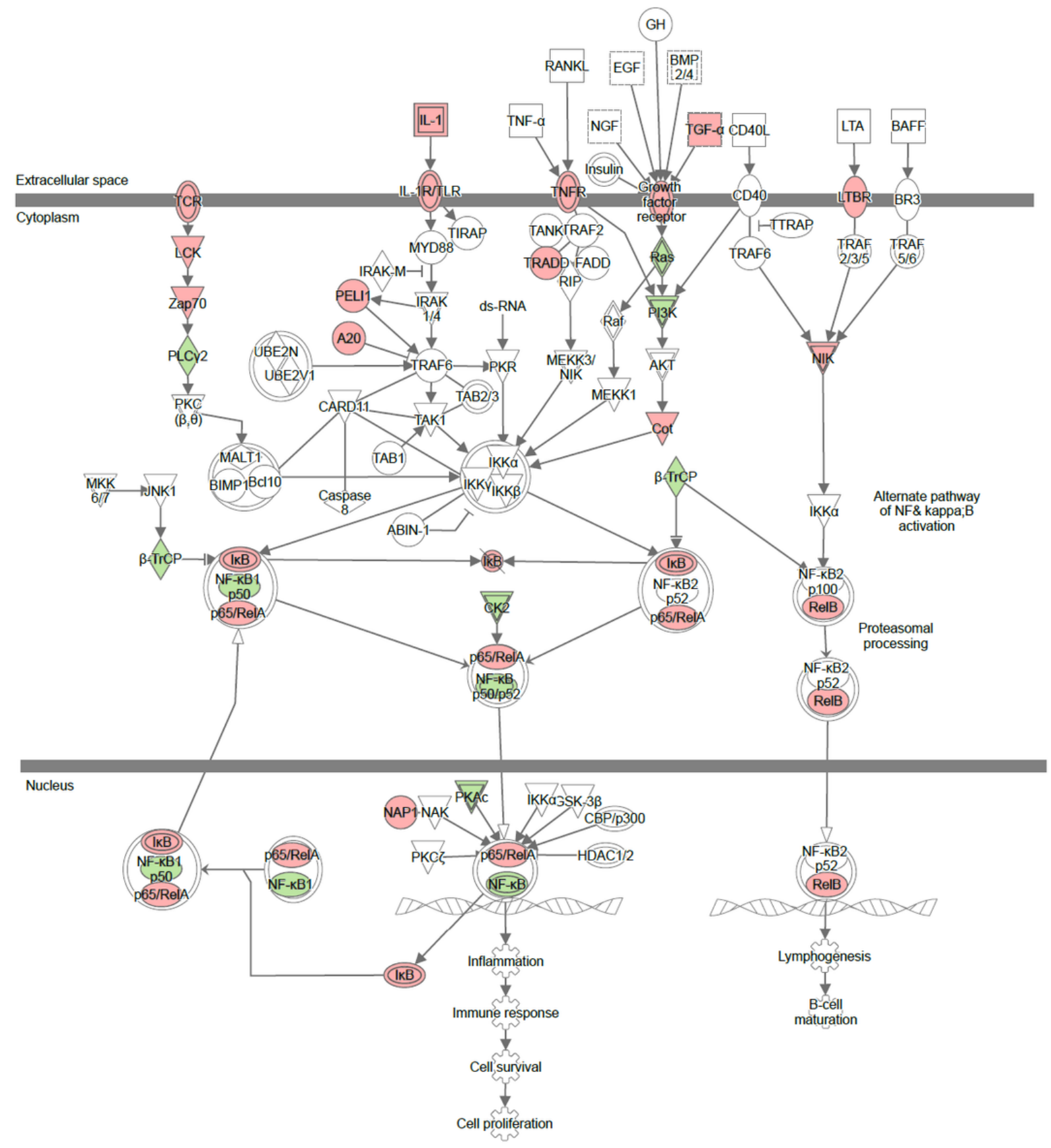

\section{Figure 1}

Activation of the NF-KB pathway. Genes marked in red are up-regulated between baseline and Day 2 while those marked in green are down-regulated. 


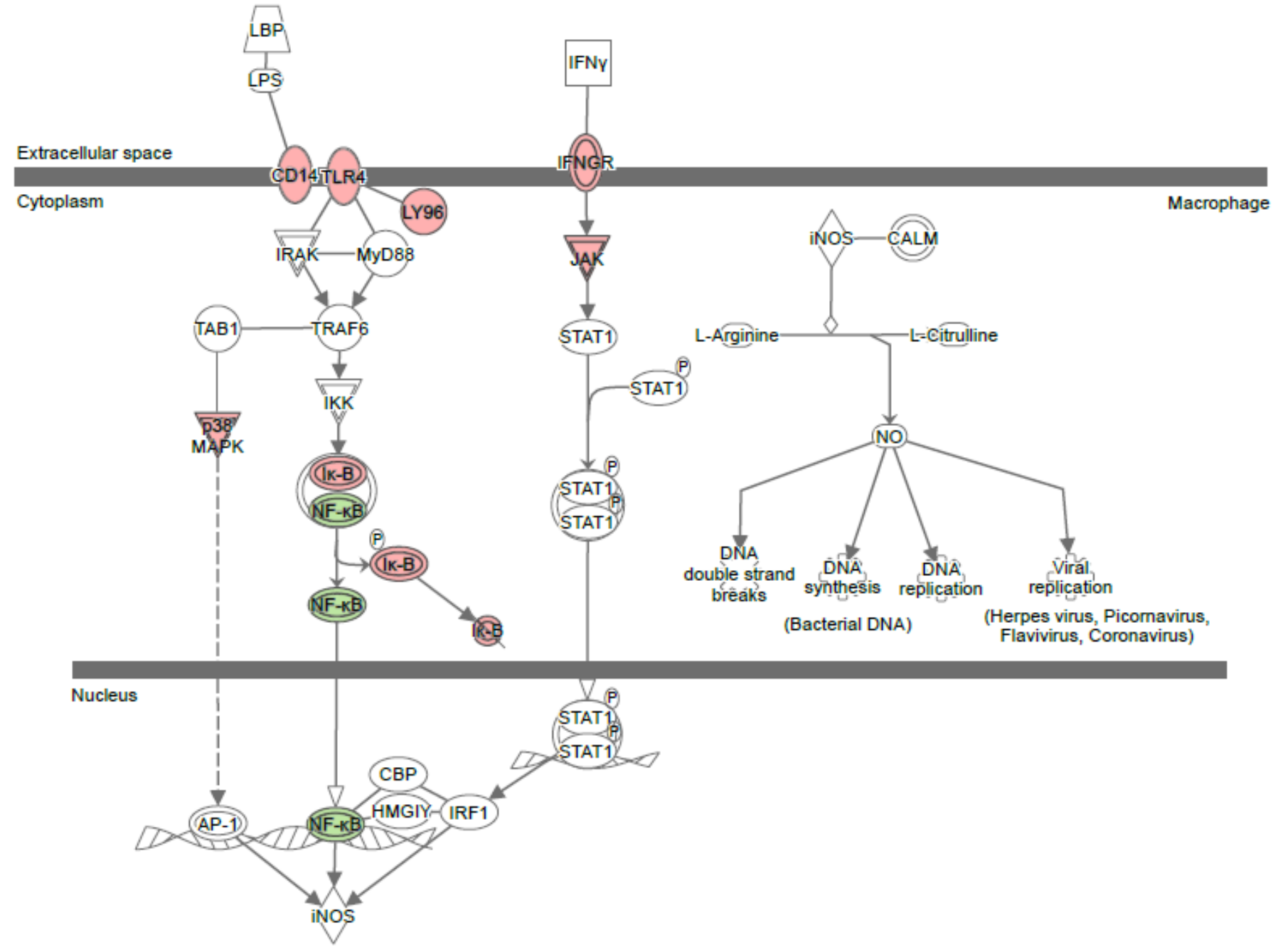

Figure 2

Activation of the iNOS pathway. Genes marked in red are upregulated between baseline and Day 2 while those marked in green are downregulated. 


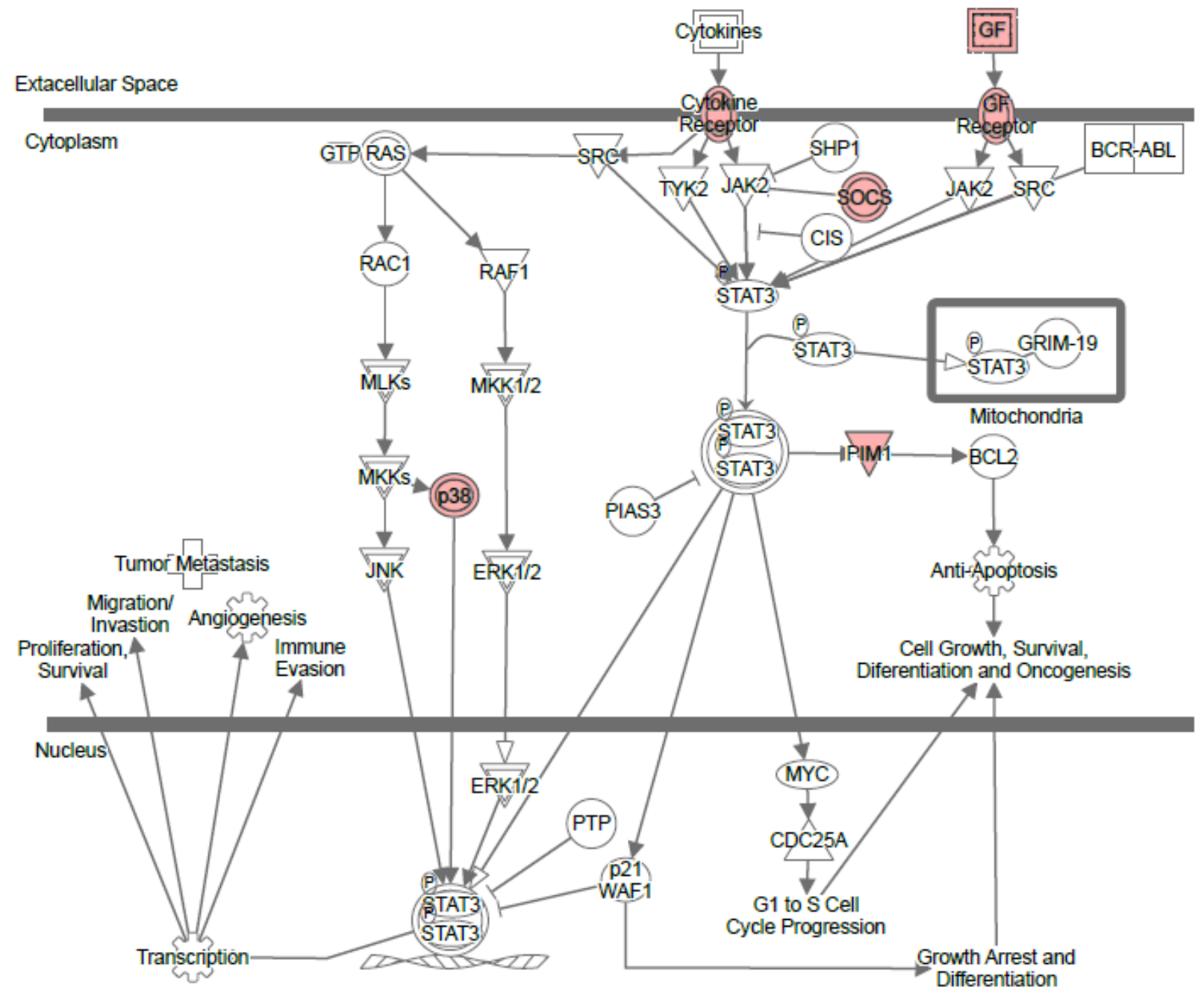

Figure 3

The STAT3 pathway. Genes marked in red are upregulated between baseline and Day 2, in patients that did not reach complete remission (CR) after the first course of induction chemotherapy. 


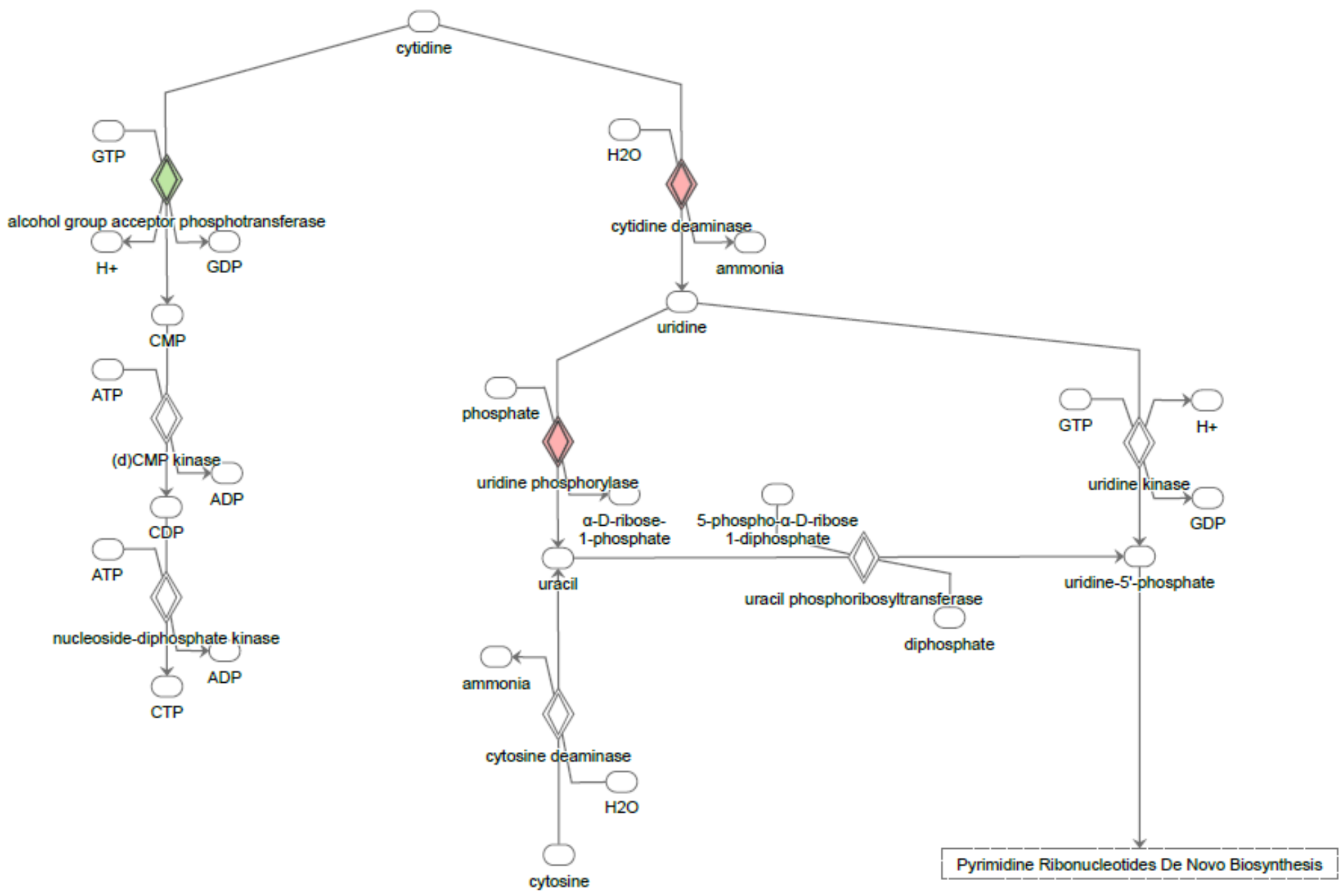

\section{Figure 4}

Salvage pathways of pyrimidine ribonucleotides. Genes marked in red are upregulated while those marked in green are downregulatedbetween baseline and Day 2, in patients that did not achieve complete remission (nCR) after the first course of induction chemotherapy. Notably, increased expression of cytidine deaminase, responsible for the deamination of Ara-C into Ara-U, could be observed.

\section{Supplementary Files}

This is a list of supplementary files associated with this preprint. Click to download.

- Additionalfile11.xlsx

- Additionalfile21.xlsx 